\title{
APLICAÇÕES DE PSEUDOSPLINES NA INTERPOLAÇÃO, SÍNTESE E ANÁLISE DE ANTENAS REFLETORAS MODELADAS
}

\author{
José Ricardo Bergmann e Flavio José Vieira Hasselmann \\ Centro de Estudos em Telecomunicações da Pontifícia Universidade Católica do Rio de Janeiro \\ Rua Marquês de São Vicente, 225, Gávea, Rio de Janeiro-RJ, 22453-900 \\ E-mail: bergmann@cetuc.puc-rio.br
}

\begin{abstract}
Resumo - O problema de interpolação global de superfícies definidas numericamente, comum na síntese e análise de antenas refletoras modeladas, é revisto com ênfase na aplicação de pseudosplines. Diversos aspectos de sua implementação são apreciados e a habilidade relativa dos principais membros daquela familia em acomodar os princípios inerentes a sínteses óticas é explorada.
\end{abstract}

Abstract - The problem of global interpolation of zumerically defined surfaces, which arises in synthesis and analysis of shaped reflector antennas, is reviewed with emphasis on the application of Pseudosplines. Several aspects of their implementation are outlined and the relative ability of pseudospline family members in accommodating the principles inherent to optical syntheses is explored.

Palavras Chave: Antenas Refletoras Modeladas, Antenas Refletoras, Interpolação por Pseudo-splines, Síntese e Análise de Antenas.

\section{INTRODUÇÃO}

O projeto de antenas refletoras para diversas aplicaçōes em telecomunicações envolve, usualmente, o emprego de écnicas de síntese das superfícies constituintes da antena, que fazem uso dos princípios da Ótica Geométrica (GO). Jesta forma, através do rastreamento da densidade de zotência ao longo de raios óticos emanados da fonte Iiimentadora e refletidos pelo refletor, obtém-se, ao se כrȩar o atendimento a características de radiação pré¿stabelecidas, uma equação diferencial parcial não-linear ¿e segunda ordem que descreve o comportamento da superfície [1]. A utilizaçāo de técnicas usuais de resolução rumérica destas equações permite, então, a obtenção de ¿m conjunto discreto de pontos que definem a superfície :efletora desejada.

Estes pontos, em geral associados a uma grade ordenada sobre um plano de referência, porém arbitrariamente distribuídos sobre a superfície refletora, devem então ser interpolados com vistas à obtenção de uma representação analítica da superfície refletora que permita o cálculo do

\footnotetext{
Este trabalho foi desenvolvido no CETUC/PUC-Rio e financiado pela Telecomunicaçōes Brasileiras S.A. através dos Contratos PUC-TELEBRÁS 414/91, 467/92 e 513/93JDPqD e pela Financiadora de Estudos e Projetos sob Convênios PUC-FINEP 56.93.0568, 56.95.0781 e 41.96.0901.
}

Publicaçāo recomendada pelo Editor de Área de Microondas e Optoeletrônica. diagrama de radiação correspondente e subseqüente verificação do atendimento às especificações de projeto. Estabelece-se, assim, um processo iterativo de síntese no qual a escolha de funções interpolantes adequadas exerce um papel importante. A utilização de funções capazes de representar a superfície de forma global e estável, apresentando a suavidade e continuidade de suas derivadas inerentes ao processo ótico de síntese e requeridas pelos métodos de análise, deve proporcionar ao projetista razoável segurança de que o não-atendimento às especificaçōes implica em refinamentos na síntese propriamente dita, descartada a ocorrência de erros associados ao processo de interpolação.

Interpolaçōes polinomiais convencionais podem introduzir oscilações entre os pontos-dados resultando em erros no cálculo do campo irradiado pelo refletor, podendo, entretanto, descrever bem, ao menos localmente, porções da superfície [2]. Splines bi-cúbicas, como utilizadas em [3] para representar superfícies refletoras definidas por pontos distribuídos em uma grade regular, fornecem superfícies suaves com derivadas contínuas até 2a. ordem, porém, como seus coeficientes variam entre células da grade, podem acarretar um tempo excessivo de procura numérica por pontos especulares em análises envolvendo traçado de raios.

Dentre as funções interpolantes globais, com as características de suavidade e continuidade acima, sāo usualmente empregadas as Séries Polinomiais de Fourier (PFS), que se mostraram adequadas para as interpolaçōes envolvidas na síntese de antenas refletoras para comunicações via satélite utilizando feixes colimados ou modelados [4,5] e a Expansão Senoidal-Polinomial de Jacobi (JPSE), descrita e aplicada em [6] na síntese de um sistema duplo-refletor com feixe colimado. Estas expansões, na forma $z(u, v)$, onde $(u, v)$ denotam coordenadas cartesianas ou cossenos diretores convenientemente normalizados, têm seus coeficientes usualmente determinados através da minimização, no sentido de mínimos quadrados, dos resíduos $\left(z-z_{j}\right)$ nos pontos-dados $\left(\mathrm{u}_{\mathrm{j}}, \mathrm{v}_{\mathrm{j}}, \mathrm{z}_{\mathrm{j}}\right)$ fornecidos pela síntese ou ainda, no caso da JPSE, fazendo uso das propriedades de ortogonalidade das funções da expansão em série. O número de termos (e coeficientes a determinar) necessário a um modelamento adequado relaciona-se às características da superfície, sendo a tolerância para os resíduos acima ditada pela convergência, dentro dos limites especificados, dos diagramas de radiação resultantes. Não-obstante, em se tratando de superfícies 
refletoras com elevado grau de modelagem, aumentar o número de termos nas expansões acima para reduzir os resíduos $\Delta \mathrm{z}$ nas porções mais críticas, afeta a descrição da superfície de uma forma global podendo, devido ao comportamento oscilatório das funções-base, deteriorar a representação de porções mais suaves. Este efeito foi verificado na implementação das PFS e JPSE para um refletor offset sintetizado para produzir um feixe em cossecante quadrada no plano vertical com irradiação azimutal uniforme, resultando uma superfície que alterna regiões com variação rápida das curvaturas em ambos os planos principais e regiões onde este comportamento se mantém apenas ao longo de uma direção principal de curvatura, observando-se o surgimento, de forma instável, de "ripples" indesejáveis nos diagramas de radiação à medida que se variava o número de termos das expansões [7].

A necessidade de se utilizar, ao menos para superfícies com alto grau de modelamento como a descrita acima, funções interpolantes globais que permitam uma implementação mais estável, levou à investigação da aplicabilidade à síntese e análise de antenas refletoras da familia de pseudosplines. Estas foram introduzidas por Duchon [8] para resolver o problema de interpolar suavemente um conjunto de pontos por uma função de duas variáveis com derivadas contínuas até certa ordem e obtidas a partir de uma extensão do problema de minimização de um funcional descritivo da energia associada a uma placa fina flexionada de modo a ocupar posições previamente definidas, tendo sido primeiramente aplicadas à interpolação de um subrefletor modelado de uma antena Cassegrain simétrica [9]. Conforme evidenciado em [7] para refletores fortemente modelados, obtém-se a convergência desejada para os diagramas de radiação utilizando-se apenas um conjunto reduzido dos pontos dados pela síntese, a partir de um algoritmo que permite refinamentos locais (pelo próprio processo de escolha daqueles pontos) em uma interpolação global e, consequientemente, evitando os problemas observados na implementaçāo de séries oscilatórias. Para antenas constituídas de superfícies mais suaves como as encontradas em sistemas refletores para feixes colimados, entretanto, o desempenho dessas expansões é comparável ao das pseudosplines [10].

A familia de pseudosplines compreende funções polinomiais de suavidade garantida por construção, com as de grau mais elevado apresentando continuidade de derivadas de mais alta ordem. Embora, ao se calcular o campo secundário da antena, seja comum integrar as correntes da Ótica Física (PO) induzidas na superfície, ao invés de utilizar métodos baseados em ótica-de-raios, o melhor atendimento aos princípios inerentes à síntese por GO deve ser buscado. Alguns destes aspectos, evidenciados em [11-13], são aqui revistos, explorandose, em particular, uma comparação entre as pseudosplines cúbicas, quínticas e de sétima ordem no que toca a representação dos parâmetros geométricos relevantes da superfície interpolada e seu efeito no cálculo do campo secundário.

\section{PSEUDOSPLINES}

Para a interpolação global de um conjunto de $\mathrm{N}$ pontos de coordenadas $\left(\mathrm{u}_{\mathrm{i}}, \mathrm{v}_{\mathrm{i}}, \mathrm{z}_{\mathrm{i}}\right)$ definindo a superfície refletora, a familia de pseudosplines, aqui restrita às cúbicas (CPS), quínticas (QPS) e de sétima ordem (SPS), é escrita [8] na forma

$$
z(u, v)=\sum_{i=1}^{N} a_{i}\left[r_{i}(u, v)\right]^{q}+\sum_{k=1}^{p} b_{k} P_{k}(u, v)
$$

com

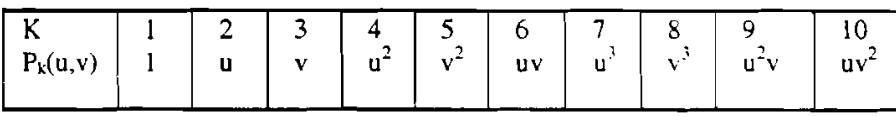

\begin{tabular}{|c|c|c|c|}
\hline & CPS & QPS & SPS \\
$Q$ & 3 & 5 & 7 \\
$P$ & 3 & 6 & 10 \\
\hline
\end{tabular}

$$
\sum_{i=1}^{N} a_{i}\left[P_{k}\left(u_{i}, v_{i}\right)\right]=0, k=1,2, \ldots . P
$$

onde

Nas expressōes acima, (u,v) refresentam $(x, y)$ ou cossenos diretores, $r_{i}(u, v)=\left[\left(u-u_{1}\right)^{2}+\left(v-u_{1}\right)^{12}\right.$ representa a distância no plano (u,v) entre o ponto onde ( $)$ ) está sendo calculada e pontos-dados de coordendas $\left(u_{1}, v_{1}\right)$; os coeficientes $\left\{a_{\mathrm{j}}\right\}_{1}^{N}$ e $\left\{b_{\mathrm{k}}\right\}_{1}^{P}$ sāo obudus pela soluçāo do sistema simétrico de $\mathrm{N}+\mathrm{p}$ equações lineares constutuído da condição de contorno (1a), imposta de forma que as derivadas contínuas de mais alta ordem permaneçam finitas afastado de cada ponto-dado. e daquelas resultantes do casamento de (1) nos $\mathrm{N}$ pontos. Em forma matricial, obtém-se:

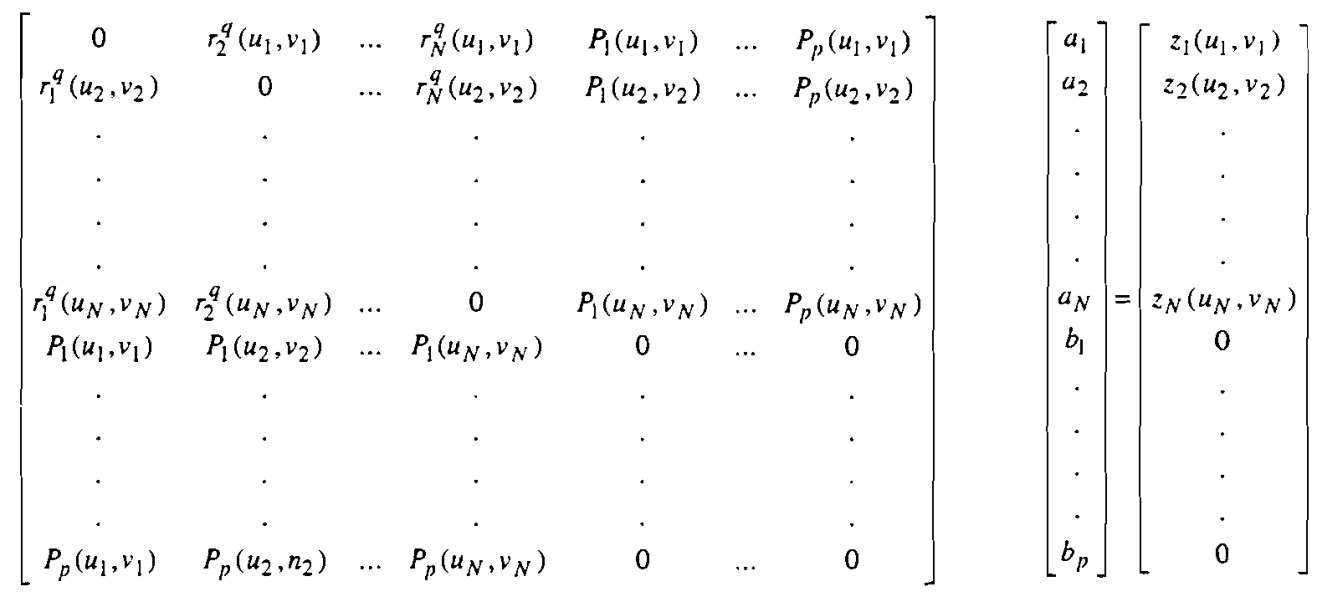


O número $\mathrm{N}$ de pontos-dados utilizados na implementação de (2) não corresponde, necessariamente, ao total de pontos fornecidos pela síntese, podendo ser apenas o suficiente para garantir a convergência do casamento sobre toda a superfície. Selecionando $\mathrm{N}_{0}\left(\mathrm{~N}_{\mathrm{o}} \geq \mathrm{p}\right)$ pontos iniciais (mais concentrados, por exemplo, nas regiōes mais críticas da superfície), adiciona-se novos pontos onde os resíduos $\Delta \mathrm{z}$ (diferença entre a coordenada $\mathrm{z}$ do ponto-dado e seu valor obtido por (1)) forem maiores, até que a tolerância desejada, associada à convergência dos diagramas de radiação correspondentes dentro das especificações, seja obtida. Neste processo iterativo, otimiza-se o esforço computacional de uma interpolação global com ações localizadas onde estas se fazem mais necessárias e resultando em um número reduzido de coeficientes a se determinar. Uma vez obtida a convergência de diagramas, fica também assegurada a convergência do algoritmo de interpolação, restando, se necessário, refinar a síntese propriamente dita.

O número de pontos (e sua localizaçāo) necessário à convergência do processo depende, naturalmente, das características da superfície, podendo também implicar na possibilidade de sintetizar menos pontos a priori como evidenciado em [7], ao contrário das expansões em série, mencionadas anteriormente, que fazem uso do conjunto completo fornecido pela síntese para minimizar os resíduos de forma global. Observa-se ainda que o valor desejado para a tolerância dos resíduos $\Delta z$, que indica o ponto de parada do algoritmo, é influenciado, de forma distinta para diferentes membros da família de pseudosplines, pela grade mais ou menos densa de pontos fornecidos pela síntese $[12,13]$. Em última análise, associa-se a tolerância desejada não só ao comportamento resultante dos parâmetros geométricos relevantes da superfície analisada mas, sobretudo, a sua influência no cálculo do campo secundário da antena, o que pode vir a permitir a convivência com tolerâncias menores $e$ conseqüente otimização do esforço envolvido. Quanto à inclusão de novos pontos-dados no algoritmo desenvolvido, há que se evitar a presença de pontos muito próximos, o que pode induzir coeficientes (da pseudospline) altos e conseqüente instabilidade numérica no compto de suas derivadas. Nestas situações, é aconselhável refinar a síntese com uma grade mais densa de forma a fornecer o grau de informação desejado sobre regiōes mais críticas da superfície, mesmo que ao custo de se utilizar mais pontos na interpolação, conforme observado no exemplo analisado em $[7,11]$.

$\mathrm{Na}$ implementação das pseudosplines a superfícies com algum grau de simetria, o número de pontos-dados necessários pode ainda ser mais reduzido. Considerando, por exemplo, superfícies representadas por $z(x, y)$ e apresentando simetria em $y$, a expressão das pseudosplines interpolantes em (1) passa a ser escrita na forma

$z(x, y)=a_{i} \sum_{i=1}^{N_{E}} a_{i}\left[r_{i}(x, y)\right]^{q}+\sum_{i=N_{E}+1}^{N_{\varepsilon}+N_{t}} a_{i}\left\{\left[r_{i}(x, y)\right]^{y}+\left[r_{i}^{\prime}(x, y)\right]^{q}\right\}+\sum_{k=1}^{p} b_{k} P_{k}(x, y)$ com

$$
\sum_{i=1}^{N_{E}+N_{S}} a_{i}\left[P_{k}\left(x_{i}, y_{i}\right)\right]=0
$$

onde

$$
\begin{aligned}
r_{i}(x, y) & \left.=\left[x-x_{i}\right)^{2}+\left(y-y_{i}\right)^{2}\right]^{1 / 2} \\
r_{i}(x, y) & =\left[\left(x-x_{i}\right)^{2}+\left(y+y_{i}\right)^{2}\right]^{1 / 2}
\end{aligned}
$$

representam, respectivamente, as distâncias entre o ponto $(\mathrm{x}, \mathrm{y})$ onde $(3)$ está sendo calculada e pontos-dados $\left(\mathrm{x}_{\mathrm{i}}, \mathrm{y}_{\mathrm{i}}\right)$ ou seus simétricos $\left(\mathrm{x}_{\mathrm{i}},-\mathrm{y}_{\mathrm{i}}\right)$, e o número total de pontosdados é dado por $\mathrm{N}=\mathrm{N}_{\mathrm{E}}+\mathrm{N}_{\mathrm{S}}$, denotando os pontos sobre o eixo de simetria $\left(N_{E}\right)$ e sobre o hemisfério $y>0\left(N_{S}\right)$. Ainda, devido à simetria, os termos de $\mathrm{P}_{\mathrm{k}}$ em potências ímpares de $y$ têm seus coeficientes $\left(b_{k}\right)$ em (3) identicamente nulos, não estando também presentes em

\begin{tabular}{|c|c|c|c|c|c|c|}
\hline $\mathrm{k}$ & $\mathrm{l}$ & 2. & 3 & 4 & 5 & 6 \\
$\mathrm{P}_{\mathrm{k}}(\mathrm{x}, \mathrm{y})$ & 1 & $\mathrm{x}$ & $\mathrm{x}^{2}$ & $\mathrm{y}^{2}$ & $\mathrm{x}^{3}$ & $\mathrm{xy}^{2}$ \\
\hline
\end{tabular}

\begin{tabular}{|c|c|c|c|}
\hline & CPS & QPS & SPS \\
$\mathrm{Q}$ & 3 & 5 & 7 \\
$\mathrm{p}$ & 2 & 4 & 6 \\
\hline
\end{tabular}

\section{(3a) e resultando:}

Como o número de pontos sobre o eixo de simetria é em geral pequeno, $N \cong N_{s}$ e, sendo $N_{s}$ a metade do número de pontos necessário em uma superfície sem simetria, o esforço computacional envolvido no casamento de (3) com a coordenada $\mathrm{z}$ dos pontos-dados tem seu tempo $\left(\alpha \mathrm{N}^{3}\right)$ aproximadamente dividido por 8. Pode-se então escrever, para o casamento em pontos $z_{j}\left(x_{j}, y_{j}\right)$ no eixo de simetria,

$$
\begin{gathered}
z_{j}\left(x_{j}, y_{j}\right)=\sum_{i=1}^{N_{E}} a_{i}\left(r_{i j}\right)^{q}+\sum_{i=N_{E}+1}^{N_{E}+N_{S}} 2 a_{i}\left(r_{i j}\right)^{q}+\sum_{k=1}^{p} b_{k} P_{k}\left(x_{j}, y_{j}\right), \\
j \leq N_{E}
\end{gathered}
$$

e, para o casamento em pontos $z_{j}\left(x_{j}, y_{j}\right)$ fora do eixo de simetria,

$$
\begin{array}{r}
z_{j}\left(x_{j}, y_{j}\right)=\sum_{i=1}^{N_{E}} a_{i}\left(r_{i j}\right)^{q}+\sum_{i=N_{E}+1}^{N_{E}+N_{S}} a_{i}\left[\left(r_{i j}\right)^{q}+\left(r_{i j}\right)^{q}\right]+\sum_{k=1}^{p} b_{k} P_{k}\left(x_{j}, y_{j}\right), N_{E} \\
<j \leq N_{E}+N_{S} \quad(4 \mathrm{~b}),
\end{array}
$$

com $r_{i j}$ e $r_{i j}$ denotando, respectivamente, as distâncias entre os pontos $\left(x_{j}, y_{j}\right)$ onde (3) está sendo calculada e os pontos-dados $\left(x_{i}, y_{i}\right)$ ou seus simétricos $\left(x_{i},-y_{i}\right)$. Em conseqüência, a equação matricial em (2) pode também ser reescrita na forma 


$\left[\begin{array}{cccc}A_{11} & A_{12} & P \\ A_{21} & A_{22} & \\ & & & 0\end{array}\right]=\left[\begin{array}{c}a_{1} \\ \cdot \\ \cdot \\ \cdot \\ a_{N} \\ b_{1} \\ \cdot \\ \cdot \\ \cdot \\ p^{T}\end{array}\right.$

onde o lado esquerdo não mais representa uma matriz simétrica, sendo constituído das matrizes $[\mathrm{P}],\left[\mathrm{P}^{\mathrm{T}}\right]$ e $[0]$ já presentes em (2) e das matrizes $A_{11}, A_{12}, A_{21}$ e $A_{22}$, de dimensões $N_{E} \times N_{E}, N_{E} \times N_{S}, N_{S} \times N_{E}$ e $N_{S} \times N_{S}$, respectivamente, e representadas por

$$
A_{11}=\left[\begin{array}{cccc}
0 & & \left(r_{i j}\right)^{q} & \\
& \cdot & & \\
& & \cdot & \\
& \left(r_{i j}\right)^{q} & & 0
\end{array}\right] \text {, }
$$

simétrica com $\mathrm{i}, \mathrm{j}$ denotando pontos sobre o eixo, e de elementos nulos na diagonal principal

$$
A_{12}=\left[2\left(r_{i j}\right)^{q}\right], \text { simétrica com } \mathrm{i} \neq \mathrm{j}
$$

$A_{21}=\left[\left(r_{i j}\right)^{q}\right] \neq A_{12}^{T}$, simétrica com $\mathrm{i} \neq \mathrm{j}$

$A_{22}=\left[\begin{array}{ccc}\cdot\left(r_{i j}\right)^{q} & + & \left(r_{i j}\right)^{q} \\ \cdot & & \\ & \left(r_{i i}\right)^{q} & \\ & & \\ \left(r_{i j}\right)^{q} & + & \left(r_{i j}\right)^{q}\end{array}\right]$, simétrica

\section{COMPARAÇÕES ENTRE CPS, QPS E SPS}

A família de pseudosplines definida em (1), incluindo as cúbicas (CPS), quinticas (QPS) e de sétima ordem (SPS) de interesse presente, constituem funções interpolantes globais, suaves por construção e apresentando derivadas contínuas de tão mais alta ordem quanto maior for sua própria ordem. Neste sentido, é razoável intuir que pseudosplines de mais alta ordem apresentem também um comportamento mais suave de suas derivadas primeira e segunda, melhor distribuido ao longo da superfície interpolada, com influência direta sobre seus parâmetros geométricos relevantes. Cumpre, então, comparar estes aspectos do desempenho das CPS, QPS e SPS levando também em conta $O$ efeito de grades mais ou menos densas de pontos fornecidos pela síntese das superfícies.

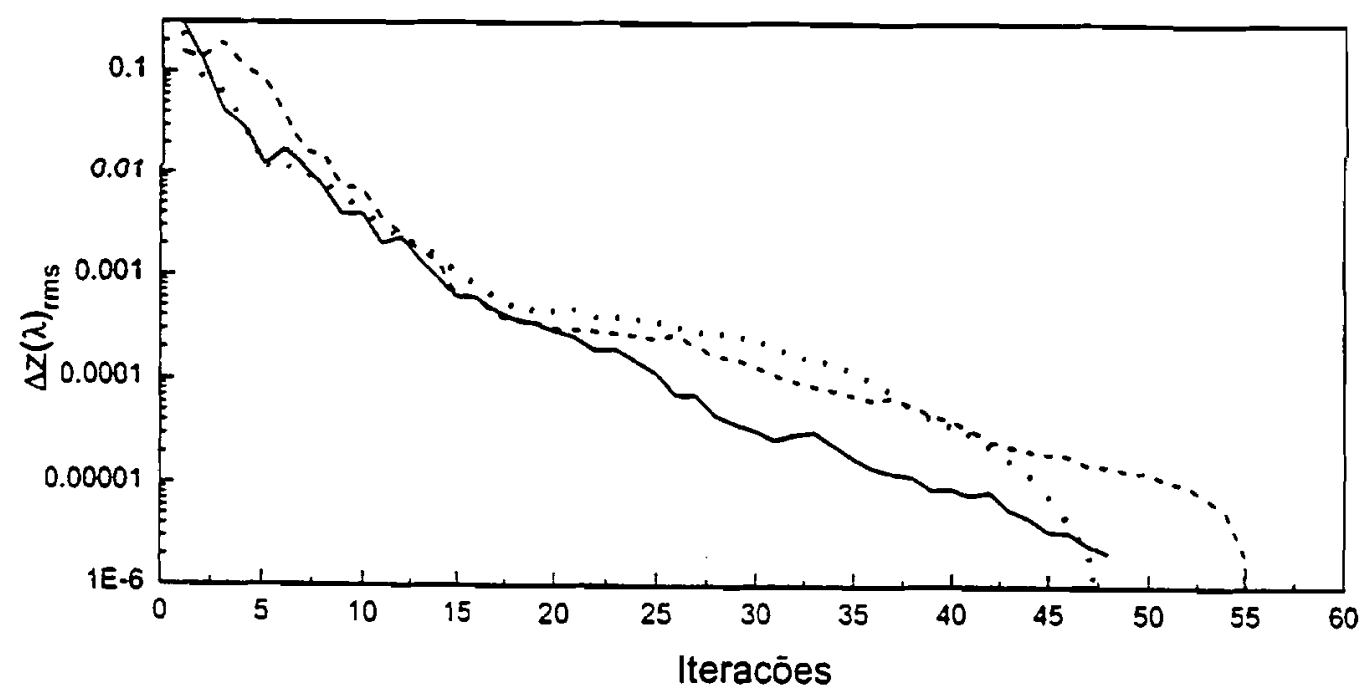

Figura 1 -. Valores rms para o erro na coordenada z para interpolação de hiperbolóide com protuberância central e espaçamento de grade $\lambda$. CPS -....-QPS SPS 
Em [12] foi analisada uma superfície com representação analítica conhecida (de forma a poder calcular os erros introduzidos pelas interpolações), posteriormente discretizada e interpolada por pseudosplines, constituída por um hiperbolóide de revolução com uma protuberância de decaimento exponencial quadrático, também simétrica e centrada no vértice do hiperbolóide na forma $z_{p}=\lambda \exp \{-$ $\left.0.15 \lambda^{-2}\left[(x-5 \lambda)^{2}+y^{2}\right]\right\}$. A partir de uma grade uniforme com espaçamento $\lambda$, mais alguns pontos intermediários na região da protuberância, a superfície foi interpolada por CPS, QPS e SPS utilizando todos os pontos (situação de erro mínimo) e, a partir do algoritmo de implementação, admitindo tolerâncias para os resíduos $\Delta z$ de $0.005 \lambda$, $0.01 \lambda$ e $0.05 \lambda$. Foram obtidas distribuições de erro $(\Delta z)$ similares para as três funções interpolantes ao longo da superfície, porém com as SPS utilizando menos pontosdados, conforme ilustrado na Fig. 1 para o valor rms de $\Delta z$ em função do número de iterações ( número de pontos dados) necessárias para convergência. Utilizando grades menos densas, com espaçamentos de $2.0 \lambda$ e $2.5 \lambda$ entre pontos, observou-se, já para a situação de erro mínimo, que as SPS apresentam menores erros com uma distribuição mais plana (suave) ao longo da superfície, especialmente na região modelada (da protuberância).

O efeito das interpolações na derivada primeira é comparado, para uma grade de espaçamento $\lambda$, através do erro absoluto na inclinação da normal à superfície calculado no plano $\mathrm{x}-\mathrm{z}$ para a situação de erro mínimo, conforme ilustrado na . $2 \mathrm{a}$; observa-se, novamente, o comportamento mais suave das SPS, especialmente com relação às CPS. Na Fig. 2b é mostrado, para as SPS, o comportamento daquele erro ao se variar as tolerâncias para $\Delta z$ no algoritmo. Observou-se também, como esperado, que o cálculo das derivadas é mais sensível à distribuição de pontos-dados utilizados pelo algoritmo de interpolação, especialmente próximo à borda da superfície, aumentando os erros envolvidos no caso de grades menos densas.

Vale ressaltar que pequenas diferenças entre as distribuiçōes de resíduos $(\Delta \mathrm{z})$ ao longo da superfície, devidas a interpolações por diferentes membros da familia de pseudosplines, não influenciam o cálculo pela $P O$ do campo secundário, visto que o algoritmo sempre converge para uma tolerância pré-especificada e a fase das correntes induzidas é acomodada apropriadamente, o mesmo acontecendo com pequenas flutuações das derivadas primeiras (e direções normais à superfície) devido à integração que se sucede [11]. No que concerne à PO, a principal diferença entre interpolações por CPS, QPS e SPS reside, portanto, no número de pontos-dados

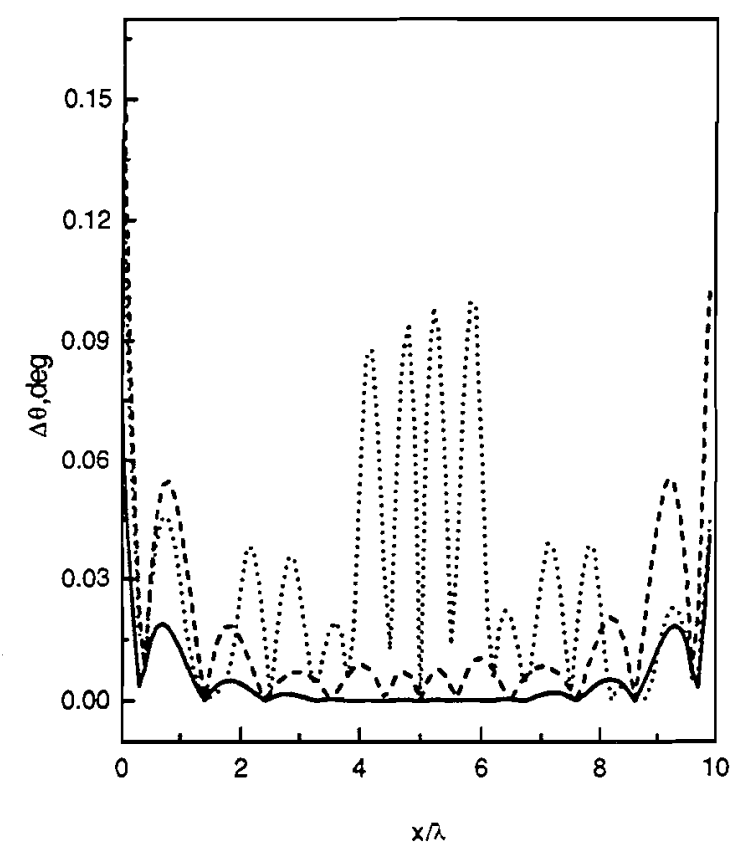

(a)

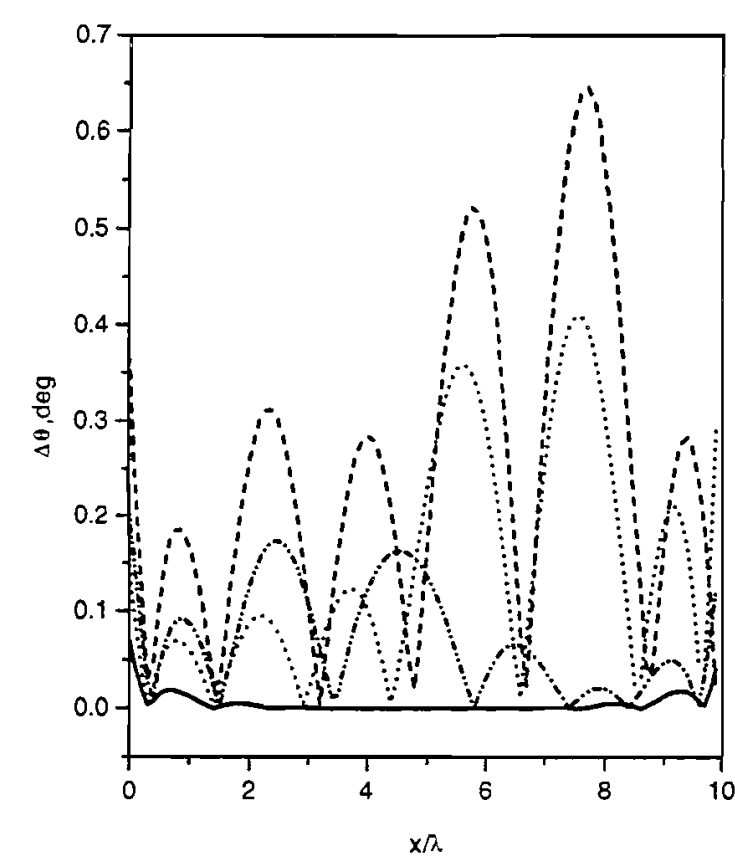

(b)

Figura 2 - Erro absoluto na direção normal ao longo do plano x-z, para interpolações de hiperbolóide com protuberância central em uma grade de espaçamento $\lambda$.

(a) Erro mínimo : ....... CPS -...-.. QPS _ SPS

(b) SPS : _ $\Delta z$ mínimo _..- $\Delta z<0.005 \lambda \ldots \Delta z<0.01 \lambda \quad \ldots \Delta z<0.05 \lambda$ 
utilizados, com implicação no número de coeficientes a serem computados para convergência do algoritmo de implementação, em especial se grades menos densas puderem ser sintetizadas. Ao se utilizar ótica-de-raios para análise, entretanto, pequenos desvios gerados por "erros" de interpolação podem afetar os diagramas obtidos pela GO e/ou o atendimento da síntese aos princípios por ela ditados.

Os erros introduzidos pelas diferentes interpolações no cálculo das derivadas de $2 \mathrm{a}$. ordem são melhor apreciados através de seu efeito sobre o cálculo do produto de suas curvaturas principais (e, consequientemente, da amplitude do campo da GO), conforme ilustrado nas Figs 3a, b, ainda referentes ao hiperbolóide com protuberância central, variando-se a tolerância de $\Delta \mathrm{z}$ para a grade de espaçamento $\lambda \mathrm{e}$ variando o espaçamento de grade para a situação de erro mínimo $\Delta z=0$ (utilizando-se todos os pontos). Observa-se novamente o excelente desempenho das SPS, embora as QPS sejam perfeitamente aceitáveis, e salienta-se a perda de suavidade, introduzida pelas CPS na região da protuberância em vista de descontinuidades nas derivadas de $3 \mathrm{a}$. ordem mais acentuadas naqueles pontosdados.

Para ilustrar os erros $\Delta G$ introduzidos por interpolações por pseudosplines na distribuição de potência irradiada por antenas refletoras segundo a GO, considerou-se a superfície analítica, analisada em [13], constituída de um elipsoide offset de distância focal $5 \lambda$, excentricidade 0.2 e diâmetro projetado $10 \lambda$, com modelamento na direção da borda superior na forma $(6 \lambda) \exp \left\{-\left(0.2 \lambda^{-2}\right)[x-\right.$ $\left.13.5 \lambda]^{2}+\left(0.01 \lambda^{-2}\right)[y]^{2}\right\}$. A superfície foi discretizada em 683 pontos distribuídos em uma grade retangular de espaçamento $0.25 \lambda \mathrm{e}$, fazendo uso da simetria no plano $x$ z, em 187 pontos para uma grade de espaçamento $0.5 \lambda$. Procedeu-se, então, a interpolações por CPS, QPS e SPS utilizando-se todos os pontos (resíduo $\Delta z=0$ ), correspondente à situação de erro mínimo, e admitindo-se tolerâncias $\Delta z=10^{-5} \lambda, 10^{-4} \lambda$ e $10^{-3} \lambda$; os números de pontos dados necessários à convergência do algoritmo são listados na Tabela 1.

Observa-se da Fig. 4a que, para interpolações por CPS, a utilização da grade de síntese mais densa $(0.25 \lambda)$ permite, para tolerâncias $(\Delta z)$ menores, a convergência do algoritmo para erros $(\Delta G)$ menores na região modelada, utilizando, porém, um número considerável de pontos dados a mais como se depreende da Tabela 1. Para interpolações por QPS, obtém-se erros $\Delta \mathrm{G}$ desprezíveis para todas as tolerâncias implementadas e para as duas grades utilizadas, conforme se observa na Fig. 4b, embora uma melhor convergência do algoritmo ao longo de toda a superfície, incluindo a região modelada, seja obtida a partir da grade mais densa. Ainda com relação às QPS, depreende-se da Tabela 1 que, não só os números de pontos-dados utilizados são menores que os correspondentes para as CPS, mas também são próximos entre si para as duas grades utilizadas, evidenciando uma maior estabilidade para as QPS. Estes efeitos são ainda mais salientados para as SPS, observando-se, da Fig. 4c, que a utilização da grade de espaçamento $0.5 \lambda$ já fornece erros $\Delta \mathrm{G}$ praticamente nulos ao longo de toda a superfície e para todas as tolerâncias. Conclui-se, então, que, no caso das SPS, pode-se utilizar resíduos maiores como tolerância, com a conseqüente redução no número de pontos-dados necessários, além de satisfazer os requisitos de sínteses óticas de forma mais suave e com convergência mais rápida do algoritmo que quando se utiliza CPS e QPS; a possibilidade de se reduzir o esforço computacional pela síntese de grades menos densas fica também evidenciada.

Para aplicaçōes envolvendo o cálculo do diagrama de radiação da antena a partir da integração das correntes da PO induzidas sobre a superfície refletora, como mencionado anteriormente, a principal diferença entre interpolações por CPS, QPS e SPS é o número de pontosdados utilizados pelo algoritmo de implementação até atingir a convergência dos diagramas correspondentes.Não só pseudosplines de ordem mais alta utilizam menos pontos como a distribuição dos pontos utilizados pelas de ordem mais baixa (CPS) tende a uma maior concentração nas regiōes mais fortemente modeladas da superfície. Discrepâncias com relaçōes às especificações de síntese, ainda que pequenas, são tipicamente observadas nos diagramas da GO devido a interpolações na borda da superfície, mas são naturalmente suplantadas nos diagramas difrativos da $\mathrm{PO}$ em diversas aplicações.

O exemplo analisado em [7], envolvendo uma superfície fortemente modelada, estabeleceu a maior estabilidade e adequação das QPS quando comparadas com a implementação de expansões em séries polinomiais/senoidais (PFS e JPSE), conforme revisto na Introdução do presente, para aquela classe de superfície. As mesmas conclusões (comparativas) se aplicaram à

\begin{tabular}{|c|c|c|c|c|}
\hline & $\Delta z=0$ & $\Delta z=10^{-5} \lambda$ & $\Delta z=10^{-4} \lambda$ & $\Delta z=10^{-3} \lambda$ \\
\hline CPS- $0.25 \lambda$ & 683 & 246 & 137 & 57 \\
\hline CPS-0.50ג & 187 & 138 & 95 & 53 \\
\hline 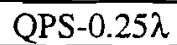 & 683 & 134 & 72 & 36 \\
\hline QPS-0.50 & 187 & 115 & 70 & 32 \\
\hline SPS- $0.25 \lambda$ & 683 & 95 & 54 & 33 \\
\hline SPS- $0.50 \lambda$ & 187 & 87 & 52 & 33 \\
\hline
\end{tabular}

Tabela 1 -.Número de pontos dados necessários para convergência de interpolações por pseudosplines de elipsóide modelado. 
implementação das CPS e SPS para aquele problema, conforme evidenciado em [11], para as CPS, e em resultados nảo publicados, para as SPS. Foi também comprovada a possibilidade de se utilizar grades de síntese menos densas. mantendo-se as características estabelecidas para a implexanzazo dis pseudosplines, enquanto as instabilidades observadas nos diagramas de radiação correspondentes às interpolações por PFS e JPSE, quando se variava o número de termos nas expansões em série, se acentuaram para sínteses formecendo distribuições mais esparsas de pontos-dados. A síntese do refletor offset modelado, analisado em $[7,11]$, forneceu uma superfície definida por 1102 pontos distribuídos ao longo de 58 radiais sobre uma grade polar regular ortogonal ao e cen-trada no eixo do alimentador. Para interpolaçōes por CPS. QPS e SPS. associadas a uma tolerância pré-especificada $\Delta z=0.005 \hat{\lambda}$ para os residuos, obteve-se convergência dos diagramas (PO) de radiação utilizando-se apenas 70, 27 e 37 pontos-dados respectivamente. Conforme esperado, pseudosplines de ordem mais alta utilizam um número inferior de pontos (e coeficientes) para convergência do algoritmo de interpolação embora se obtenha uma concordância satisfatória entre os diagramas da PO, conforme ilustrado ia Fig.5.

\section{Referências}

.1] Westcott, B., "Shaped Reflector Antenna Design", Research Studies Press, Letchworth, Herts, UK, 1983.

.2] Mehler, M., Tun, S. e Adatia, N., "Direct far-field GO synthesis of shaped beam reflector antennas", Proc. IEE, vol. 133, pt. H, no. 3, pp. 213-220, 1986.

¿] Lee, S.W., Cramer Jr., P., Woo, K. e Rahmat-Samii, Y., "Diffraction by an arbitrary subreflector: GTD solution", IEEE Trans. Antennas Propagat., vol. AP27, no. 3, pp. 305-316, 1979.

i] Clarricoats, P.J.B., Bergmann, J.R. e Brown, R.C., "Synthesis of reflector antenna radiation patterns", URSI Electromag. Theory Symp. Digest, pp. 325326,1983

5] Bergmann, J.R., Brown, R.C., Clarricoats, P.J.B. e Zhou, H., "Synthesis of shaped-beam reflector antenna patterns", Proc. IEE, vol. 135, pt. H. no. 1, pp. $48-53,1988$.

6] Galindo-Israel, V., Imbriale, W. A., Rahmat-Samii, Y. e Veruttipong, T., "Interpolation methods for shaped reflector analysis", IEEE Trans. Antennas Propagat., vol. AP-36, no. 3, pp. 441-444, 1988.

7] Bergmann, J.R., Hasselmann, F.J.V., Teixeira, F.L. e Rego, C.G., "A comparison between techniques for global surface interpolation in shaped reflector analysis", IEEE Trans. Antennas Propagat., vol. AP42, no, 1, pp. 47-53, 1994.
[8] Duchon, J., "Interpolation des fonctions de deux variables suivant le principe de la flexion des plaques minces", R.A.I.R.O. Analyse Numérique, vol. 10, no. 12, pp. 5-12, 1976.

[9] Pereira, L.C.P. e Hasselmann, F.J.V., “Approximation of shaped reflector surfaces by pseudosplines", IEEE AP-S Int. Symp. Digest, vol. 1, pp. 327-329, 1984.

[10] Bergmann, J.R., Hasselmann, F.J.V., Costa, L.P.N., Teixeira, F.L. e Rego, C.G., "On the interpolation of numerically defined surfaces in collimated-beam dual reflector antennas", Microwave Opt. Technol. Lett. vol. 7, no. 4, pp. 209-212, 1994.

[11] Bergmann, J.R. e Hasselmann, F.J.V., "Interpolation of shaped reflector surfaces by cubic pseudosplines", Microwave Opt. Technol. Lett., vol. 8, no. 5, pp. 247-250, 1995 .

[12] Bergmann, J.R. e Hasselmann, F.J.V., "Modelling surface raw data for reflector antenna applications", Proceedings of IEE International Conference on Computation in Electromagnetics, pp. 405-410, 1996.

[13] Bergmann, J.R. e Hasselmann, F.J.V., "On the interpolation of reflector surfaces by pseudosplines", IEE Proc. Microwaves, Antennas and Propagat., vol. 144, no.1, pp. 42-46, 1997. 


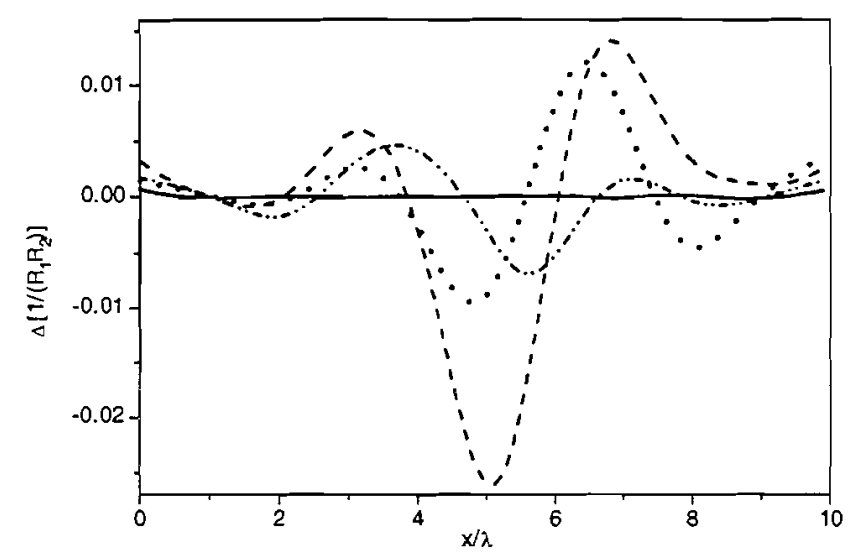

(iii)

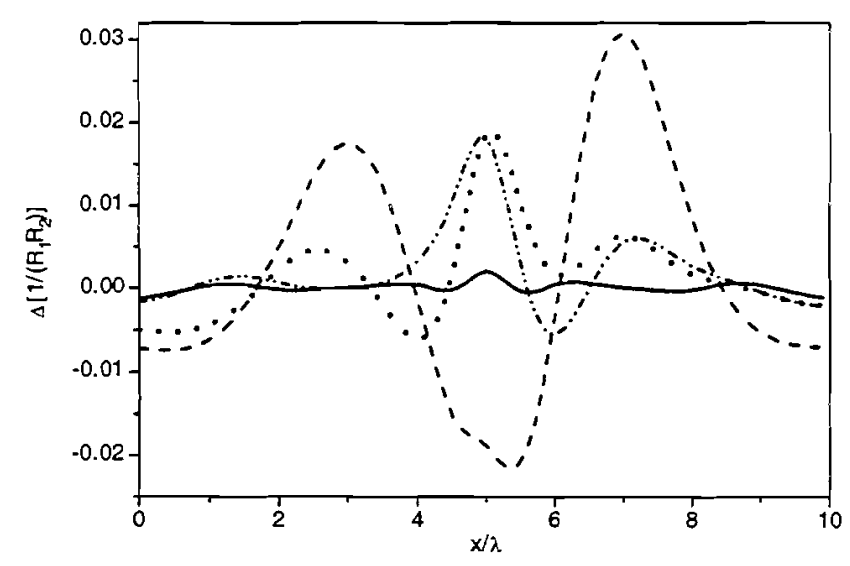

(ii)

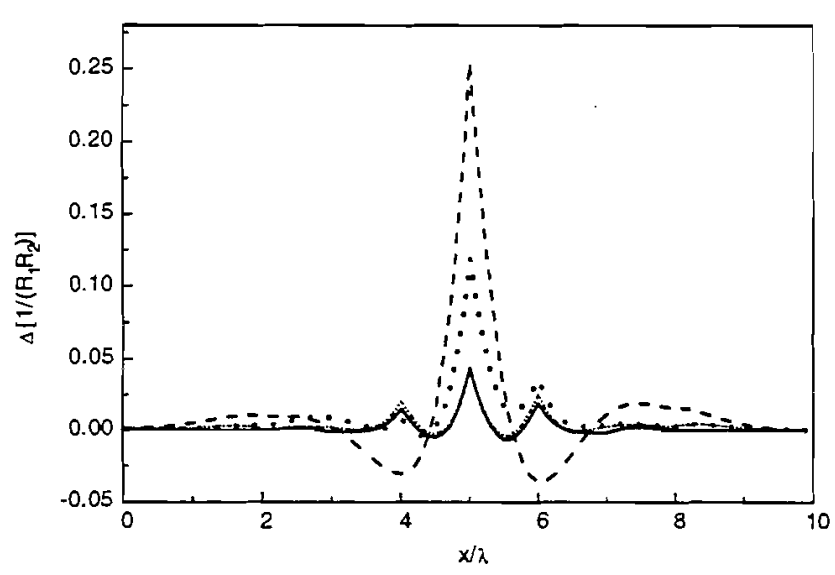

(i)

(a)

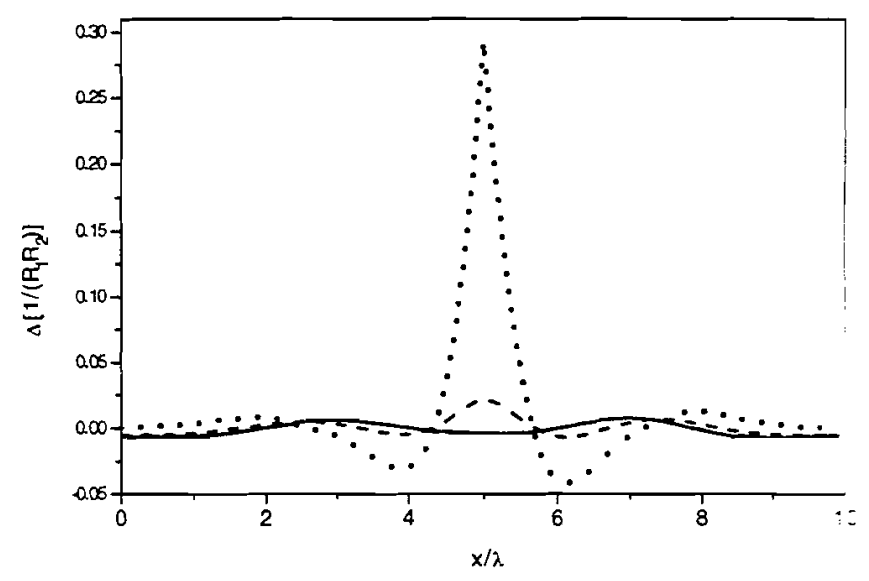

(iii)

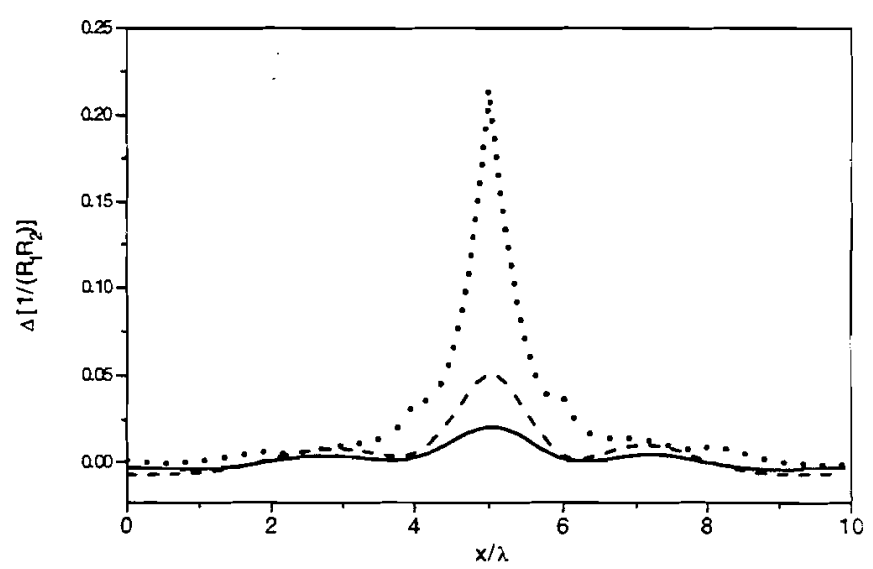

(ii)

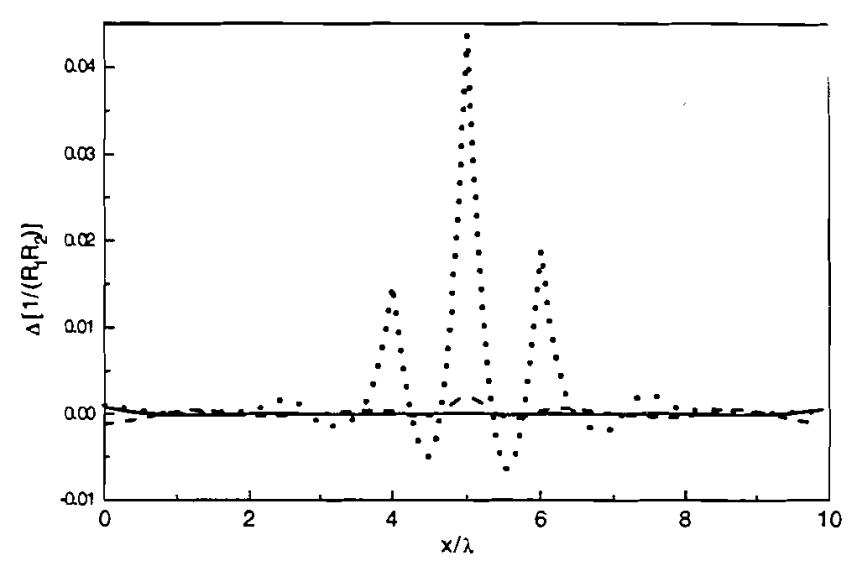

(i)

(b)

Figura 3 - Erro no produto das curvaturas principais ao longo do plano $x \cdot z$ para interpolações por pseudosplines de hiperbolóide com protuberância central.

(a) espaçamento • de grade : (i) CPS, (ii) QPS, (iii) SPS

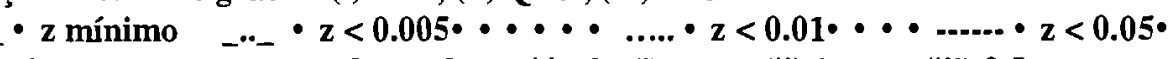

(b) erro mínimo e espaçamento de grade variável : (i) $\cdots$ (ii) $2 \cdots$ (iii) $2.5 \bullet$ 

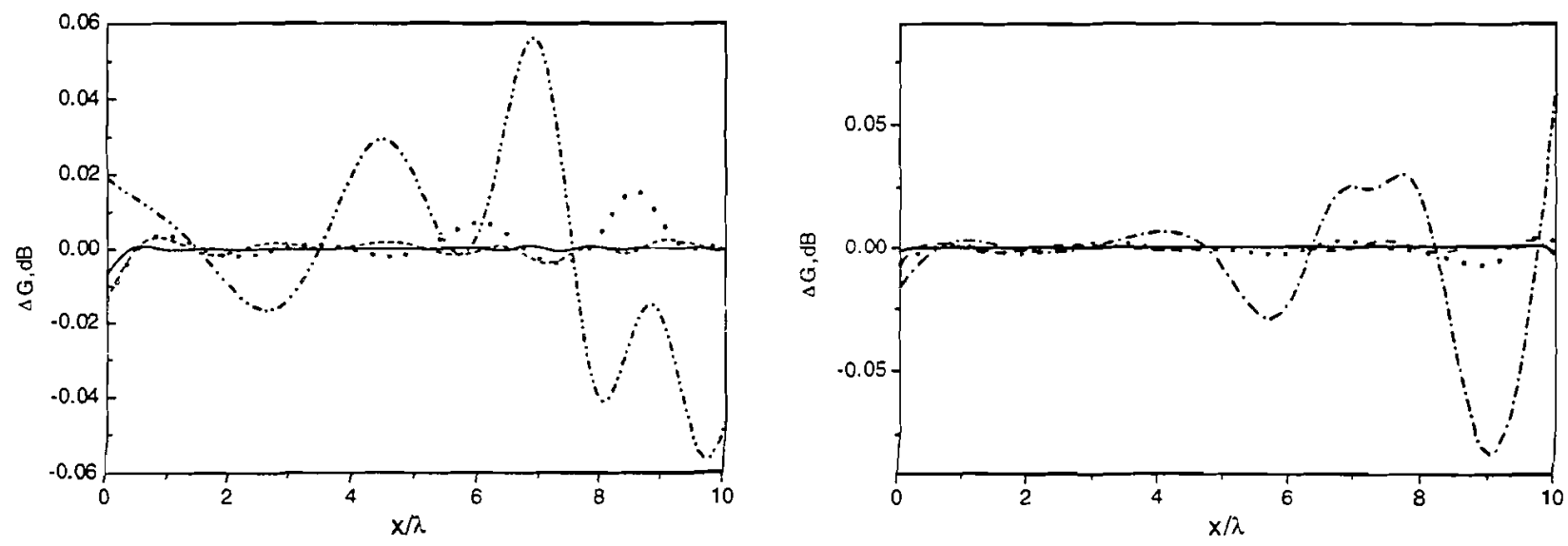

(i)

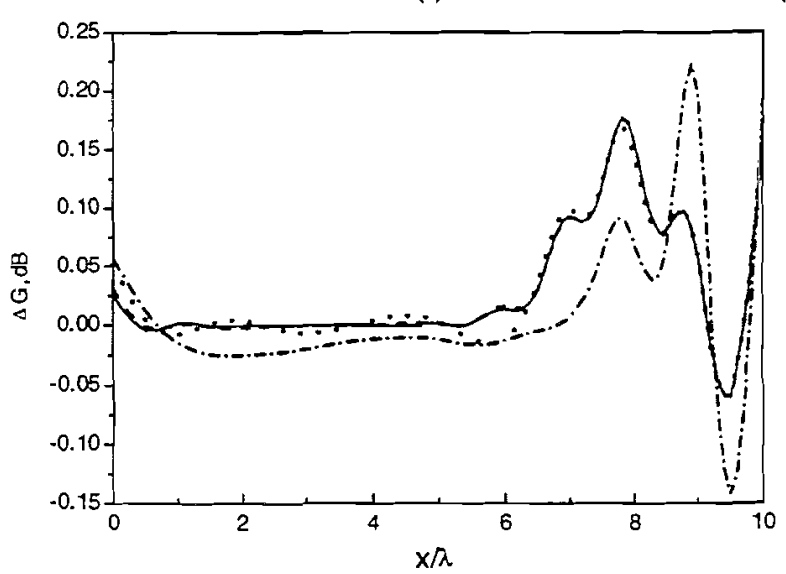

(i)

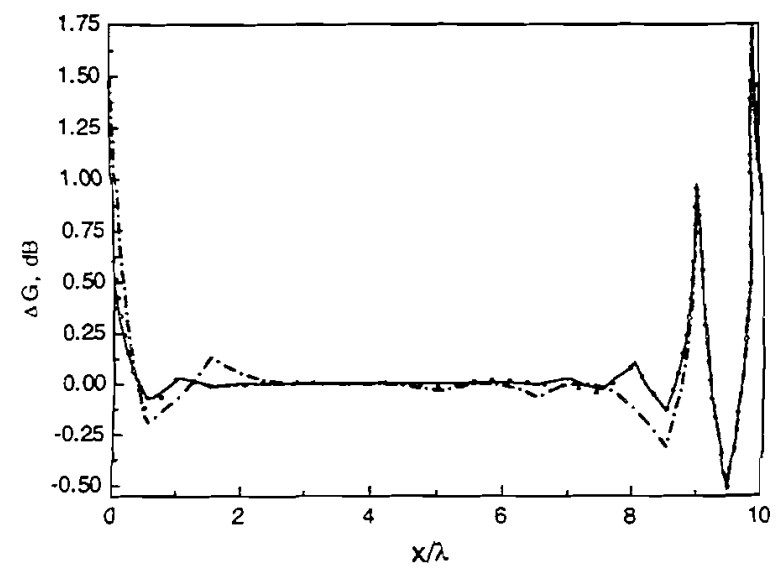

(i) (c) SPS

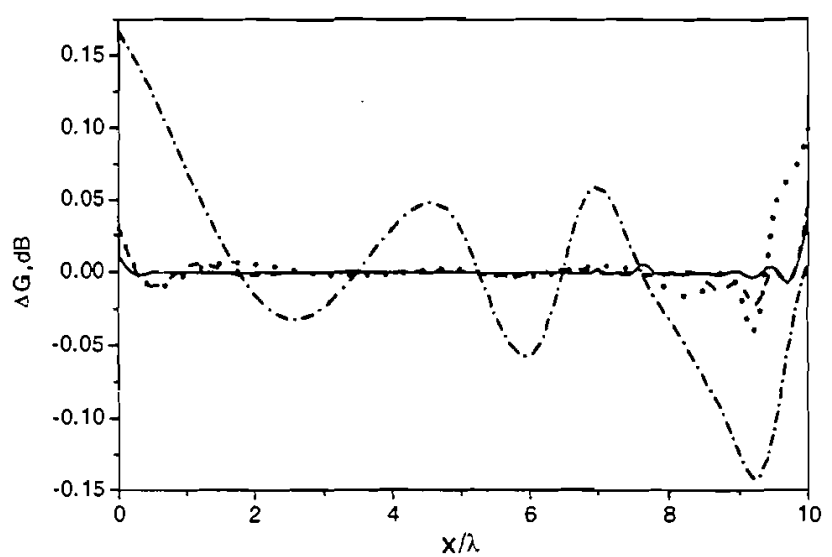

(b) QPS

(ii)

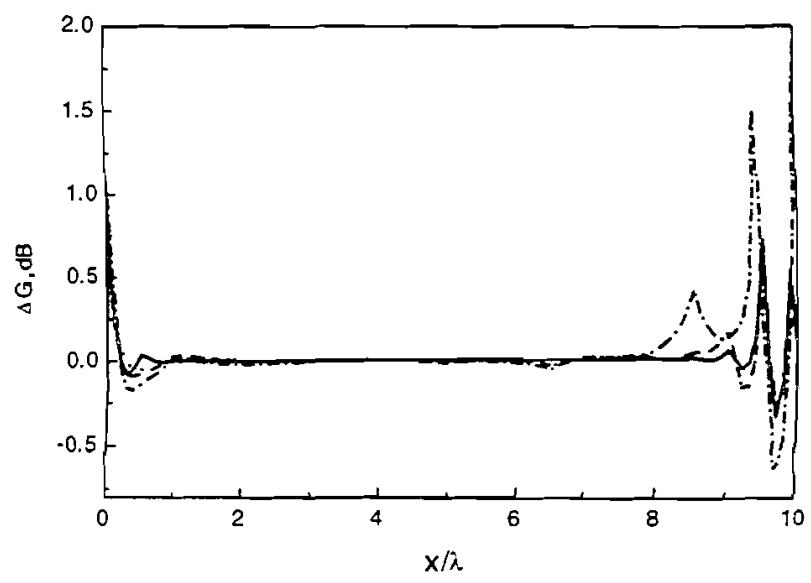

(ii)

Figura 4 - Erro na distribuição de potência (GO) irradiada no plano x-z, relacionada aos pontos de reflexão, por elipsóide offset modelado para interpolações por pseudosplines a partir de espaçamentos de grade (i) $0.5 \lambda$, (ii) $0.25 \lambda$

$$
\Delta z=0 \cdots \Delta z=10^{-5} \lambda \ldots \Delta z=10^{-4} \lambda \ldots \Delta z=10^{-3} \lambda
$$




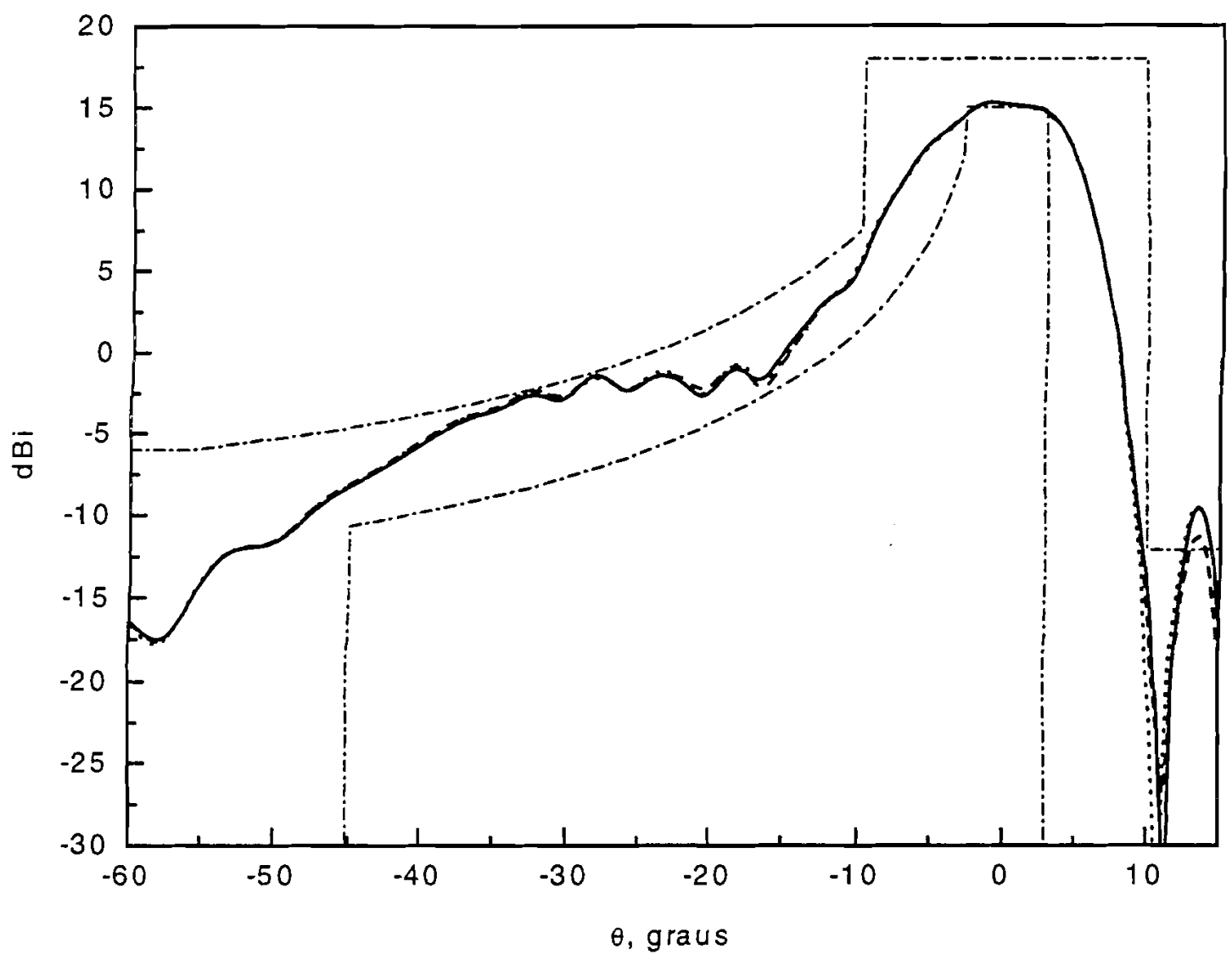

Figura 5 - Diagramas de radiação (PO), no plano de simetria, de refletor modelado interpolado por pseudosplines com tolerâncias segundo a Tabela 2.

-_-_- especificações de projeto SPS $\quad \ldots .$. QPS ..CPS

José Ricardo Bergmann nasceu em Porto Alegre em 25 de março de 1953. Graduou-se em Engenharia Elétrica pela Universidade Federal do Rio Grande do Sul em dezembro de 1975. Recebeu o título de Mestre em Engenharia Elétrica (Msc) pelo Instituto Militar de Engenharia em fevereiro de 1979 e o título de Doutor em Engenharia Elétrica ( $\mathrm{PhD}$ ) pela University of London em dezembro de 1986. É professor associado do Centro de Estudos em Telecomunicações e coordenador de pósgraduação do Departamento de Engenharia Elétrica da Pontifícia Universidade Católica do Rio de Janeiro, estando vinculado a esta universidade desde 1979. Seus principais interesses em pesquisa são eletromagnetismo aplicado, síntese e análise de antenas refletoras e antenas para comunicações via satélite. É atualmente chairman do Capítulo Conjunto Rio de Janeiro das Sociedades AP/MTT/ED do IEEE.
Flavio José Vieira Hasselmann obteve os diplomas de Engenheiro Eletricista (Telecomunicações) e Mestre em Ciências em Engenharia Elétrica pela Pontifícia Universidade Católica do Rio de Janeiro em 1974 e 1976, respectivamente, e de Doctor of Phylosophy (Electrophysics) pelo Polytechnic Institute of New York (hoje, Polytechnic University) em 1980. É professor associado do CETUC (Centro de Estudos em Telecomunicações da PUC/Rio), de onde foi vice-diretor no periodo de 1984 a 1996, e vice-chairman do Capítulo Conjunto Rio de Janeiro das Sociedades AP/MTT/ED do IEEE. Na SBT, foi membro do Conselho Deliberativo no período 1983-1985, coordenador técnico do IV Simpósio Brasileiro de Telecomunicações e editor da área de antenas e propagação da Revista da SBT no período 1987-1991. Seus principais interesses em pesquisa são em métodos assintóticos em eletromagnetismo e antenas refletoras. 